\title{
$\alpha$-synuclein and tyrosine hydroxylase expression in acute rotenone toxicity
}

\author{
CAN LUO ${ }^{1}$, ALI H. RAJPUT ${ }^{1,2,3}$, SHAKEEL AKHTAR ${ }^{1}$ and ALEXANDER RAJPUT ${ }^{1,2,3}$ \\ ${ }^{1}$ Saskatchewan Centre for Parkinson's Disease and Movement Disorders; ${ }^{2}$ Division of Neurology, \\ Department of Medicine, University of Saskatchewan; ${ }^{3}$ Saskatoon Health Region, Saskatoon, Saskatchewan, Canada
}

Received September 7, 2006; Accepted October 24, 2006

\begin{abstract}
Chronic low-dose (2-3 mg/kg/day) rotenone infusion produces clinical features and biological markers of Parkinson's disease (PD) in some rats. A significant proportion of rats, however, die of acute rotenone toxicity. Most studies have focused on chronic rotenone-infused rats. It has not been established if the animals that die of acute low-dose rotenone toxicity manifest clinical or pathological evidence of PD. In the present study, six rats that received continuous $3 \mathrm{mg} / \mathrm{kg} /$ day subcutaneous rotenone infusion, became moribund and were euthanized after five days were compared with ten vehicle infused animals sacrificed 14, 28 or 56 days after placebo infusion. All rotenone-infused rats had significant motor function decline beginning one day after the infusion and progressive worsening in the physical condition until they became severely akinetic, at which point they were euthanized. In the substantia nigra of rotenone-treated rats, four of six had reduced numbers of tyrosine hydroxylase-positive neurons and all six had increased nigral $\alpha$-synuclein expression. Our observations show that even a short duration of low-dose subcutaneous rotenone infusion can induce clinical and pathological markers of PD in some rats. The pathophysiology of the enhanced susceptibility to PD in some animals remains to be established.
\end{abstract}

\section{Introduction}

Parkinson's syndrome (PS) is characterized by a combination of bradykinesia, rigidity and tremor. The most common pathological variant of PS is Parkinson's disease (PD) (1-3) which is characterized by marked loss of substantia nigra (SN) dopaminergic neurons and Lewy body inclusions. The

Correspondence to: Dr Alexander H. Rajput, Division of Neurology, Department of Medicine, Room 1659 Royal University Hospital, University of Saskatchewan, 103 Hospital Drive, Saskatoon, SK, S7N 0W8, Canada

E-mail: rajputa@sask.usask.ca

Key words: rotenone, Parkinson's disease, $\alpha$-synuclein, tyrosine hydroxylase, toxicity
Lewy bodies (LB) are rich in $\alpha$-synuclein (4). Mutation of $\alpha$-synuclein is known to produce Parkinsonian features and LB pathology $(5,6)$. The loss of dopaminergic neurons and formation of LB inclusions do not go hand in hand as evident by absence of Lewy bodies in 1-methyl-4-phenyl-1, 2, 3, 6 tetrahydropyridine (MPTP) exposed human subjects that have marked loss of SN neurons $(5,7,8)$. Rotenone is a lipophilic compound found in many plants of the leguminosae family, which are widely distributed in the world. After chronic infusion with rotenone, some rats develop clinical features of PD (9). These animals show reduced TH immunoreactivity in the $\mathrm{SN}$ and $\alpha$-synuclein-positive inclusions in the SN neurons $(9,10)$. There are, however, wide variations in the level of rotenone toxicity in rats. In one study $36 \%$ of the rotenoneinfused animals died within the first three days while others survived for a long time (10). Studies of PD markers have so far focused on chronic rotenone-infused animals (9-12). To our knowledge there is no information on pathological markers of PD in the animals that die of low-dose rotenone toxicity during the first week of infusion. We report on PD markers in rats that suffered from acute rotenone toxicity on low-dose subcutaneous infusion.

\section{Materials and methods}

Animals and treatment. All procedures involving animals were in strict accordance with guidelines established by the Canadian Council on Animal Care, and approved by the University of Saskatchewan Animal Care Committee.

Male Lewis rats, weighing between 344-396 g at the time of surgery, were randomly divided into those that received vehicle (placebo) or rotenone infusion. Alzet osmotic minipumps (model 2ML4, Durect Corporation, Cupertino, CA) (10) were aseptically filled with rotenone dissolved in equal amounts of dimethyl sulfoxide (DMSO) and polyethylene glycol (PEG) (all from Sigma, St. Louis, MO) or with DMSO/ PEG vehicle for implantation. The animals were anesthetized with $5 \%$ isoflurane in pure oxygen and maintained on 1-2\% isoflurane in oxygen during surgery. The minipump was placed subcutaneously in the back using an aseptic technique. Rotenone-treated animals were continuously infused with $3 \mathrm{mg} / \mathrm{kg}$ per day (calculated on weight at the time of surgery) and placebo animals received DMSO/PEG at the same rate of fluid infusion as the rotenone-treated rats. Each animal was housed separately in a cage and maintained on a 12-h light 
and dark cycle with free access to food and water. In rotenonetreated animals that did not die or require euthanizing, and the control animals, the pump was replaced with a new pump containing the same fluid on the 29th day (the day when the first surgery was performed is day 0 ). Throughout the treatment the animals were monitored daily for weight loss and for motor manifestations. During the first 3 weeks they were weighed daily and subsequently every 3 days. When an animal lost more than $5 \%$ of the pre-treatment weight, it was given food supplement Ensure (Ross Laboratories, OH, USA) 5-8 ml and/or lactated Ringer's USP solution $10 \mathrm{ml}$ twice daily. When further deterioration occurred such that the animal could not be fed adequately and was unable to ambulate, the institutional guidelines required discontinuing the dietary supplement and the animal was euthanized (13).

Before sacrifice the animal was deeply anesthetized with sodium pentobarbital (Abbott Laboratories, Montreal, Canada), $40 \mathrm{mg} / \mathrm{kg}$, i.p. and was sacrificed by decapitation. The brain was removed immediately and cut into two equal halves along the longitudinal fissure. The left half was stored at $-70^{\circ} \mathrm{C}$ and the right half was fixed in $4 \%$ paraformaldehyde in $0.1 \mathrm{M}$ phosphate-buffered saline (PBS, $\mathrm{pH} 7.4$ ) for 2 days and then cryoprotected in $30 \%$ sucrose for $2-3$ days at $4^{\circ} \mathrm{C}$. Finally, the right half of the brain was frozen in 2-methylbutane prechilled with dry ice and then stored at $-70^{\circ} \mathrm{C}$ until further analysis. The brains from the rotenone-infused rats and those from the placebo-infused rats were processed identically.

Immunohistochemistry. Coronal sections of the striatum $(40 \mu \mathrm{m})$ and the substantia nigra $(30 \mu \mathrm{m})$ were cut on a freezing microtome (Leica SM 2000R) and collected in 24well plates containing $0.01 \mathrm{M}$ PBS. The detection of TH and $\alpha$-synuclein immunoreactivity was performed using conventional avidin-biotin-immunoperoxide technique as described previously with minor modifications (9). Freefloating sections were rinsed three times in 0.01 M PBS and then pretreated with $0.3 \%$ hydrogen peroxide in PBS for $30 \mathrm{~min}$ to eliminate endogenous peroxidase activity. After rinsing three times in $0.01 \mathrm{M} \mathrm{PBS}$, the sections were preincubated at room temperature for $1 \mathrm{~h}$ with a blocking solution containing 5\% normal horse or goat serum and $0.3 \%$ Triton $\mathrm{X}-100$ in $0.01 \mathrm{M}$ PBS. They were then incubated at $4^{\circ} \mathrm{C}$ with monoclonal mouse anti-TH (1:1000, Chemicon, Temecula, CA, USA) or polyclonal rabbit anti- $\alpha$-synuclein (1:1000, Sigma) for $48 \mathrm{~h}$ and then at room temperature for an additional $2 \mathrm{~h}$. The primary antibodies were localized with a Vectastain ABC kit (Vector Laboratories, Burlingame, CA, USA). The reaction product was visualized with the development solution containing $0.03 \% \mathrm{DAB}$ and $0.03 \%$ hydrogen peroxide in $0.01 \mathrm{M}$ PBS ( $\mathrm{pH} 7.4$ ) for 5-10 min. The sections were mounted onto polylysine-coated slides, air dried, dehydrated, cleared, and coverslipped. For the purpose of immunohistochemical control we performed the procedures as above except we omitted the primary antibodies.

Quantification of dopaminergic neurons in the substantia nigra. Every 6th section (from bregma -4.8 to $-6.04 \mathrm{~mm}$ ) of the substantia nigra was immunostained for TH (e.g. section $1,7,13$ ) and for $\alpha$-synuclein (e.g. section $2,8,14$ ). The quantification of $\mathrm{TH}$-positive neurons in the substantia nigra compacta $(\mathrm{SNc})$ was performed with stereological protocol (14). TH-positive neurons in every 6th section of SNc were counted. The number of TH-positive neurons counted in all sections was multiplied by 6 to obtain the total number of TH positive neurons in SNc. The counting was performed with a x40 objective.

Densitometric analysis. Every 6th section (from bregma 2.2 to $-0.26 \mathrm{~mm}$ ) of the striatum was immunostained for $\mathrm{TH}$ or for $\alpha$-synuclein. Digital images were obtained using an Olympus BH2-RFCA microscope fitted with a Spot-RT digital camera (Diagnostic Instruments, Sterling Heights, MI) and analyzed using Image-Pro Plus software (Media Cybernetics, Silver Spring, MD). The measured areas of the striatum (target areas) were determined as follows. The outer border of the striatum was defined by the lateral ventricle medially and by the corpus callosum dorsally and laterally. The areas of the corpus callosum were measured as the areas of background. The measured areas of the substantia nigra (target areas) were throughout the substantia nigra $(\mathrm{SN})$. Peduncle areas were measured as the areas of background. With Image-Pro Plus software, the data were obtained as a gray-scale value of the measured area; i.e. the bigger the number the darker the staining, the smaller the number the lighter the staining. The following formula was used to calculate the percentage difference score (DS) in optical density (15):

$$
\text { Target gray scale - Background gray scale }
$$

$$
\overline{(\text { Target gray scale }+ \text { Background gray scale }) / 2}
$$

Clinical features. All rats were videotaped in a round area of $40-\mathrm{cm}$ diameter for $2 \mathrm{~min}$ on the day before implanting the pump. After minipump implantation, video tapes were made on the first day, every third day subsequently and on the day of sacrifice or euthanasia. The clinical features were analyzed based on those videotapes.

Statistics. Rotenone-treated and placebo rats were compared using Student's t-test. All analyses were carried out using SPSS software (13.0 for Windows, 2004). The level of significance was set at $\mathrm{P}<0.05$.

\section{Results}

Selection of animals for the study. Fifty animals received rotenone and 17 received placebo infusion. Four (8\%) of the rotenone-treated animals were found dead during the first week and 14 others (28\%) became moribund and required euthanizing during the first week. Of the latter 14 rats, six were randomly chosen for this study. Ten placebo-treated rats randomly chosen for sacrifice, three after 14 days, three after 28 days and four after 56 days, were used in this study.

Clinical symptoms of the rotenone-treated rats. All placebotreated rats remained healthy after minipump implantation throughout the course of follow up. All six rotenone-treated rats included in this study manifested motor function impairment including unsteady ambulation $(\mathrm{n}=5)$, walking backwards $(n=4)$, slowed mobility $(n=4)$, hunched back posture $(n=4)$, and circling movement $(n=3)$. Some motor impairment was evident one day after infusion which became worse until the 

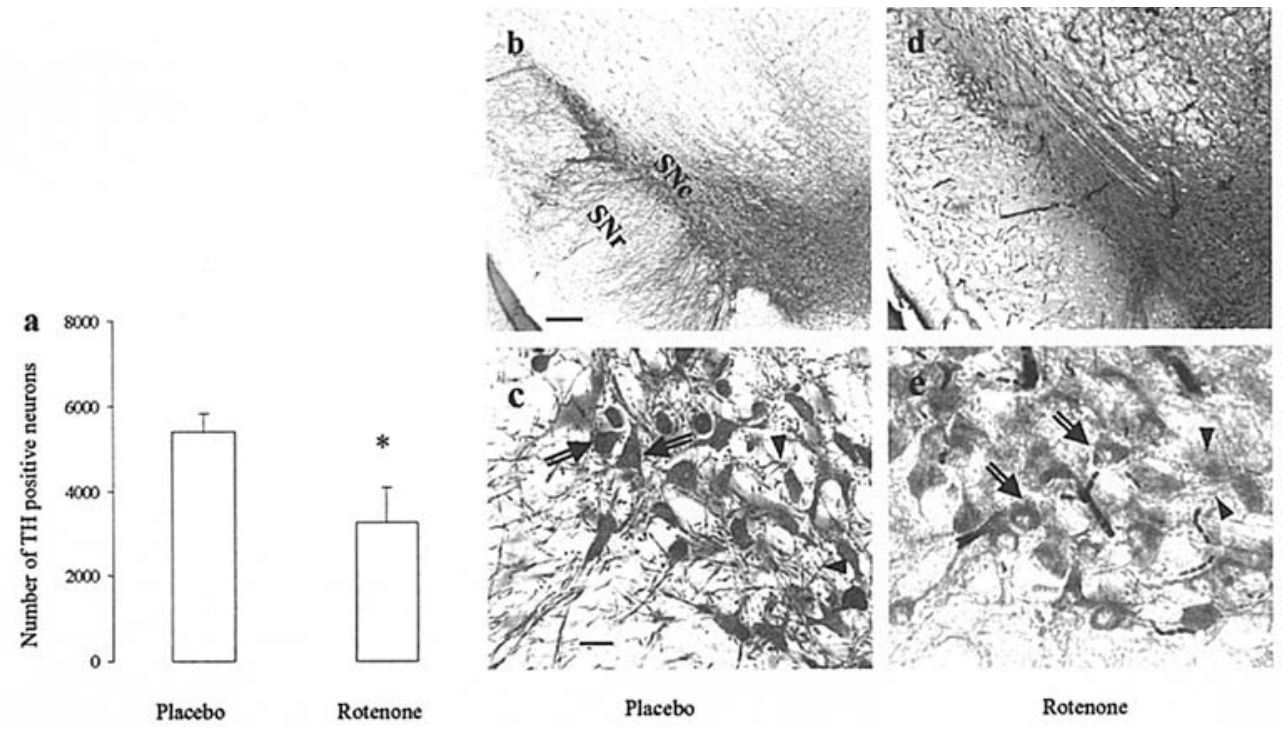

Figure 1. Tyrosine hydroxylase (TH)-positive neurons and fibers in substantia nigra in controls and rotenone-treated animals. Comparison between placebo $(\mathrm{n}=10)$ and rotenone $(\mathrm{n}=6)$ groups revealed a significantly reduced number of TH-positive neurons in SN pars compacta (SNc) in the rotenone-treated group $(\mathrm{P}<0.05)$ (a). The data is expressed as mean $\pm \mathrm{SE}$. Representative photographs are shown in b-e. In placebo rats, TH-positive neurons in SNc and abundant fine $\mathrm{TH}$-positive fibers in substantia nigra pars reticulata ( $\mathrm{SNr}$ ) (b). The outline and processes of TH-positive neurons (indicated by arrows) and neurites (indicated by arrowheads) among neurons were observed (c). (d and e) From rotenone-infused rats. The number of TH-positive neurons in SNc is reduced and TH-positive fibers in SNr are not visible (d). The neurons (indicated by arrows) showed lower intensity of TH immunostaining and indistinct outlines. The debris of neuronal and neurite lysis (indicated by arrowheads) was observed (e). Scale bar=200 $\mu \mathrm{m}$ (b) and $20 \mu \mathrm{m}$ (c).
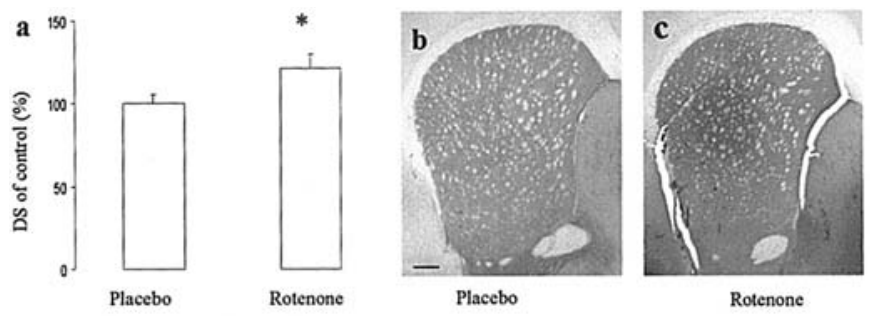

Figure 2. Effect of acute rotenone toxicity on the level of $\mathrm{TH}$ in striatum. Comparison between placebo $(n=10)$ and rotenone-treated $(n=6)$ groups revealed significantly increased levels of $\mathrm{TH}$ in striatum in the rotenone-treated group $(\mathrm{P}<0.05)(\mathrm{a})$. The data is expressed as mean $\pm \mathrm{SE}$. Representative photographs are shown in $\mathrm{b}$ and $\mathrm{c}$. The density of $\mathrm{TH}$ immunostaining in rotenone-treated rats (c) was higher than that in placebo rats (b) and the round area with the darkest staining was also observed (c). Scale bar, $400 \mu \mathrm{m}$ (b).

rats were severely akinetic and had to be euthanized five days after rotenone infusion. In four of the six rats between the second and fifth day, there was bleeding from the nose and/or hemorrhages in the eyelids.

Rotenone reduced TH-IR neurons in the SNc. In rotenonetreated rats, the number of $\mathrm{TH}$-positive neurons in the $\mathrm{SNc}$ was reduced to approximately $60 \%$ that of controls $(\mathrm{P}<0.05)$ (Fig. 1a). The representative photographs of SN sections of rats are shown in Fig. 1b-e. In the placebo-treated rats, THpositive neurons were concentrated in the SNc and dense, fine TH-IR fibers were observed in the substantia nigra pars reticulata (SNr) (Fig. 1b). The outline of the SNc neurons was clear and TH-IR neurites were observed interspersed between them (Fig. 1c). In rotenone-treated rats, the TH-positive neurons were significantly reduced in SNc and the fine TH-IR positive fibers were not visible in the $\mathrm{SNr}$ (Fig. 1d). Additionally, the
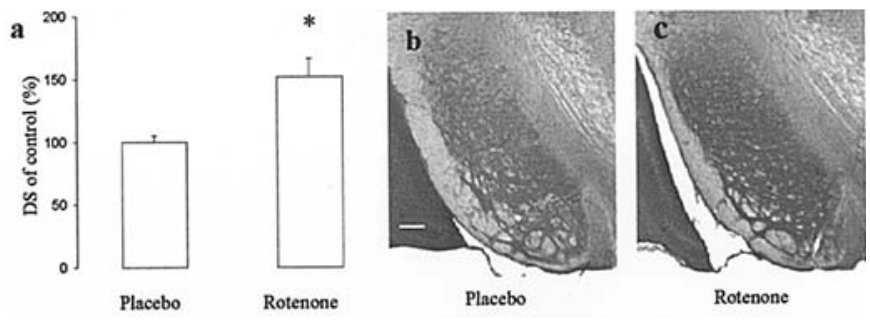

Figure 3. Effect of acute rotenone toxicity on the level of $\alpha$-synuclein in SNr. Comparison between placebo $(n=10)$ and rotenone-treated $(n=6)$ groups revealed significantly increased levels of $\alpha$-synuclein in $\mathrm{SNr}$ in the rotenone-treated group $(\mathrm{P}<0.05)$ (a). The data is expressed as mean $\pm \mathrm{SE}$. Representative photographs are shown in b and c. The density of $\alpha$-synuclein immunostaining in rotenone-treated rats (c) was higher than that in placebo rats (b). Scale bar, $250 \mu \mathrm{m}$ (b).

outline of the TH-positive neurons was indistinct, there was loss of TH-IR neurites and TH staining was reduced in the SNc neurons (Fig. 1e).

Effect of rotenone on TH immunostaining density in the striatum. The rotenone-treated rats as a group had increased striatal TH immunostaining density (Fig. 2a). Representative photographs are shown in Fig. $2 \mathrm{~b}$ and c. In the rotenonetreated animals, the TH staining density in the striatum was higher than that in the placebo group (Fig. 2b and c).

Rotenone increased $\alpha$-synuclein immunostaining density in the substantia nigra. $\alpha$-synuclein immunostaining density in the $\mathrm{SN}$ was significantly increased in the rotenone-infused rats (Fig. 3a). Fig. 3b shows the $\alpha$-synuclein immunoreactivity in the placebo-treated rats. Fig. 3c shows $\alpha$-synuclein staining in the rotenone-treated rats. 


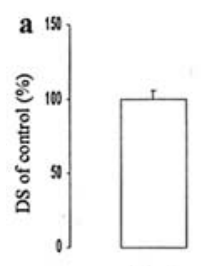

Placebo

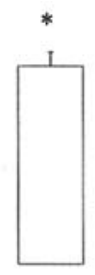

Rotenone
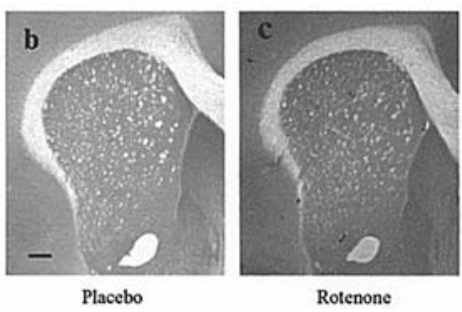

Figure 4. Effect of acute toxicity on the level of $\alpha$-synuclein in striatum. Comparison between placebo $(n=10)$ and rotenone-treated $(n=6)$ groups revealed significantly increased levels of $\alpha$-synuclein in striatum in the rotenone-treated group $(\mathrm{P}<0.05)(\mathrm{a})$. The data is expressed as mean $\pm \mathrm{SE}$. Representative photographs are shown in $b$ and $c$. The density of $\alpha$-synuclein immunostaining in rotenone-treated rats (c) was higher than that in placebo rats (b). Scale bar, $0.8 \mathrm{~mm}(\mathrm{~b})$.

Rotenone increased $\alpha$-synuclein staining density in the striatum. Rotenone-infused rats had significantly increased $\alpha$-synuclein immunostaining density in the striatum (Fig. 4a). Representative photographs are shown in Fig. $4 b$ and c. The immunostaining density was higher in rotenone-treated rats (Fig. 4c) than the control rats (Fig. 4b). Depite increased immunostaining there was no aggregation of $\alpha$-synuclein into Lewy bodies.

\section{Discussion}

All ten rats infused with vehicle placebo remained healthy throughout the study. By contrast, all six rotenone-infused rats included in the study had impaired motor function resembling PD features that manifested one day after infusion followed by progressive motor decline requiring euthanizing during the first week. Thus the motor disability was attributable to rotenone and not to the insertion of the pump or the vehicle used to dissolve rotenone.

High-dose rotenone $(10-18 \mathrm{mg} / \mathrm{kg} / \mathrm{day})$ infusion for one week has been reported to produce neuronal loss in the striatum and globus pallidus, but the $\mathrm{SN}$ neurons were spared (16). Individual variations in the response to rotenone infusion have been well-studied in rats $(8-10,12)$, but the reasons for them remain unknown. In chronic low dose (2-3 mg/kg/day) rotenone infusion TH-IR neuronal loss in the $\mathrm{SN}$ has been reported in several studies $(9-11,17,18)$. While some reports indicate selective dopaminergic degeneration and $\alpha$-synuclein aggregation (10), others found more widespread pathological changes comparable to multiple system degeneration (18).

To our knowledge, there is no literature on PD markers in animals that died early on low-dose $(2-3 \mathrm{mg} / \mathrm{kg} /$ day $)$ rotenone infusion. We restricted the study to only those rats in which we could identify the time of death (euthanizing) precisely and excluded the rats that died naturally at an unknown time. The two major findings in our rats were: i) the damage to the TH-IR neurons; and ii) the increased $\alpha$-synuclein expression in the substantia nigra. The substantia nigra TH-positive neurons manifested some degree of pathology in every rotenone-infused animal in our study. The majority (four of six) had marked loss of TH-positive SNc neurons and reduced number of TH-positive fibers (Fig. 1d and e). The two rotenone-treated animals that had normal TH-positive neuronal count also demonstrated pathological evidence characterized by swollen neurons and reduced number of TH-positive fibers (photograph not shown). The loss of TH-positive neurons in the SNc paralleled the loss of TH-positive neuropil in the SNr. Whether the dysfunction of the neurons resulted in antegrade degeneration of the neuropil or the damage to neurofibers resulted in retrograde degeneration of neurons as suggested by some studies $(9,12)$ cannot be resolved by the present study.

Chronic rotenone toxicity may show no reduction in TH-IR immunostaining, patchy focal loss or diffuse loss of TH-IR fibers in the striatum $(10,12,19,20)$. We noted similar variations in striatal $\mathrm{TH}$ staining. The four rotenone-treated rats that had lost neurons in the SNc showed no elevation in striatal TH staining compared with placebo animals, though the TH staining was increased in rotenone-treated animals as a group. We observed circumscribed areas with higher TH staining in the striatum of an animal that had normal THpositive neuronal count in SNc (Fig. 2c). The reasons for these variations remain to be elucidated.

Partial damage to the nigrostriatal dopamine (DA) system can cause acute loss of DA innervation and induce compensations for that loss in the striatum (21). Compensatory responses include the increase of $\mathrm{TH}$ synthesis, increase of DA release and turnover, and decrease of DA uptake (21). In the present study, however, there was no change downstream in the striatal TH immunostaining when there was loss of nigral neurons.

Lewy body (LB) inclusions are considered as the hallmark of Parkinson's disease pathology $(5,22-24)$. $\alpha$-synuclein is the major component of Lewy bodies and Lewy neurites $(4,25)$. Chronic low-dose rotenone infusion can produce $\alpha$-synuclein aggregation in SN neurons (9-11) and in vitro studies show increased $\alpha$-synuclein expression in human dopaminergic (SH-SY5Y) cells after 24-h rotenone exposure (26). We evaluated $\alpha$-synuclein expression with immunohistochemical quantification, which revealed increased immunostaining for $\alpha$-synuclein in both the substantia nigra ( $\mathrm{SNr}$ ) and striatum in all rotenone-treated animals that became moribund and were sacrificed within one week of infusion. However, $\alpha$-synuclein aggregation, i.e. Lewy body formation, was not found in nigral neurons in our study. The pathophysiological significance of $\alpha$-synuclein aggregation remains controversial $(27,28)$. Three possibilities have been postulated regarding $\alpha$-synuclein aggregation: i) it is directly toxic to the cell; ii) it is an inert marker of an underlying metabolic defect; or iii) it is a protective reaction (28). Increased $\alpha$-synuclein staining without aggregation may be a precursor for Lewy body formation. The true significance of increased $\alpha$-synuclein staining with or without aggregation remains to be determined.

\section{Acknowledgements}

We are grateful to Dr M.K. Pasha who helped with animal experiments. We wish to thank Curling Classic and P.W. Golf classic for their financial support and also Dr Xin-Min Li for allowing the use of his laboratory equipment.

\section{References}

1. Jellinger K: The pathology of Parkinsonism. In: Movement Disorders 2. Marsden CD and Fahn S (eds). Butterworths and Corp., London, pp124-165, 1987. 
2. Rajput AH, Rozdilsky B and Rajput A: Accuracy of clinical diagnosis in Parkinsonism - A prospective study. Can J Neurol Sci 18: 275-278, 1991.

3. Rajput AH, Rozdilsky B, Rajput A and Ang L: Levodopa efficacy and pathological basis of Parkinson syndrome. Clin Neuropharmacol 13: 553-558, 1990.

4. Spillantini MG, Schmidt ML, Lee VM, Trojanowski JQ and Jakes R: Alphasynuclein in Lewy bodies. Nature 388: 839-840, 1997.

5. Hardy J and Lees AJ: Parkinson's disease: A broken nosology. Mov Disord 20 (suppl 12): S2-S4, 2005.

6. Polymeropoulos MH, Lavedan C, Leroy E, Ide SE, Dehejia A, Dutra A, Pike B, Root H, Rubenstein J, Boyer R, Stenroos ES Chandrasekharappa S, Athanassiadou A, Papapetropoulos T, Johnson WG, Lazzarini AM, Duvoisin RC, Di Iorio G, Golbe LI and Nussbaum RL: Mutation in the alpha-synuclein gene identified in families with Parkinson's disease. Science 276: 2045-2047, 1997.

7. Langston JW, Forno LS, Tetrud J, Reeves AG, Kaplan JA and Karluk D: Evidence of active nerve cell degeneration in the substantia nigra of humans years after 1-methyl-4-phenyl1,2,3,6-tetrahydropyridine exposure. Ann Neurol 46: 598-605, 1999.

8. Perier C, Bove J, Vila M and Przedborski S: The rotenone model of Parkinson's disease. Trends Neurosci 26: 345-346, 2003.

9. Betarbet R, Sherer TB, MacKenzie G, Garcia-Osuna M, Panov AV and Greenamyre JT: Chronic systemic pesticide exposure reproduces features of Parkinson's disease. Nat Neurosci 3: 1301-1306, 2000.

10. Sherer TB, Kim JH, Betarbe R and Greenamyre JT: Subcutaneous rotenone exposure causes highly selective dopaminergic degeneration and alpha-synuclein aggregation. Exp Neurol 179: 9-16, 2003.

11. Hoglinger GU, Feger J, Prigent A, Michel PP, Parain K, Champy P, Ruberg M, Oerte WH and Hirsch EC: Chronic system complex I inhibition induces a hypokinetic multisystem degeneration in rats. J Neurochem 84: 491-502, 2003.

12. Zhu C, Vourc'h P, Fernagut PO, Fleming SM, Lacan S, Dicarlo CD, Seaman RL and Chesselet MF: Variable effects of chronic subcutaneous administration of rotenone on striatal histology. J Comp Neurol 478: 418-426, 2004.

13. Pasha MK, Sharma RK and Rajput AH: Increased myocardial N-myristoyltransferase activity in rotenone model of Parkinsonism. Int J Mol Med 15: 987-991, 2005.

14. Malberg JE, Eisch AJ, Nestler IJ and Duman RS: Chronic antidepressant treatment increases neurogenesis in adult rat hippocampus. J Neurosci 20: 9104-9110, 2000.
15. Luo $\mathrm{C}, \mathrm{Xu} \mathrm{H}$ and $\mathrm{Li} \mathrm{XM}$ : Post-stress changes in BDNF and Bcl-2 immunoreactivities in hippocampal neurons - Effect of chronic administration of olanzapine. Brain Res 1025: 194-202, 2004.

16. Ferrante RJ, Schulz JB, Kowall NW and Beal MF: Systemic administration of rotenone produces selective damage in the striatum and globus pallidus, but not in the substantia nigra. Brain Res 753: 157-162, 1997.

17. He Y, Imam SZ, Dong Z, Jankovic J, Ali SF, Appel SH and Le W: Role of nitric oxide in rotenone-induced nigro-striatal injury. J Neurochem 86: 1338-1345, 2003.

18. Hoglinger GU, Lannuzel A, Khondike ME, Michel PP, Duyckaerts C, Fege J, Champy P, Prigent A, Medja F, Lombes A, Oertel WH and Hirsch EC: The mitochondrial complex I inhibitor rotenone triggers a cerebral tauopathy. J Neurochem 95: 930-939, 2005

19. Milusheva E, Baranyi M, Kittel A, Sperlagh B and Vizi ES: Increased sensitivity of striatal dopamine release to $\mathrm{H}_{2} \mathrm{O}_{2}$ upon chronic rotenone treatment. Free Radic Biol Med 39: 133-142, 2005.

20. Caboni P, Sherer TB, Zhang N, Taylor G, Na HM, Greenamyre JT and Casida JE: Rotenone, deguelin, their metabolites, and the rat model of Parkinson's disease. Chem Res Toxicol 17: 1540-1548, 2004.

21. Song DD and Haber SN: Striatal responses to partial dopaminergic lesion: evidence for compensatory sprouting. J Neurosci 20: 5102-5114, 2000 .

22. Duvoisin RC and Golbe LI: Toward a definition of Parkinson's disease. Neurology 39: 746, 1989.

23. Eriksen JL, Wszolek Z and Petrucelli L: Molecular pathogenesis of Parkinson disease. Arch Neurol 62: 353-357, 2005.

24. Gibb WRG and Lees AJ: The relevance of the Lewy body to the pathogenesis of idiopathic Parkinson's disease. J Neurol Neurosurg Psychiatry 51: 745-752, 1988.

25. Spillantini MG, Crowther RA, Jakes R, Hasegawa M and Goedert M: $\alpha$-synuclein in filamentous inclusions of Lewy bodies from Parkinson's disease and dementia with Lewy bodies. Proc Natl Acad Sci USA 95: 6469-6473, 1998.

26. Watabe $M$ and Nakaki T: Rotenone induces apoptosis via activation of bad in human dopaminergic SH-SY5Y cells. J Pharmacol Exp Ther 311: 948-953, 2004.

27. Perez RG and Hastings TG: Could a loss of alpha-synuclein function put dopaminergic neurons at risk? J Neurochem 89: 1318-1324, 2004

28. Tofaris GK, and Spillantini MG: Alpha-synuclein dysfunction in Lewy body diseases. Mov Disord 20 (suppl 12): S37-S44, 2005 . 